\title{
Structure of the Human 26S Proteasome Revealed by Cryo-Electron Microsocpy
}

\author{
Paula C.A. da Fonseca* and Edward P. Morris ${ }^{*}$ \\ ${ }^{*}$ Structural Electron Microscopy Team, Section of Structural Biology, The Institute of Cancer \\ Research, London, SW3 6JB, UK.
}

In eukaryotes the ubiquitin/proteasome pathway is responsible for the controlled targeting and degradation of a wide range of proteins, including key cellular regulators such as those controlling cell cycle progression and apoptosis. The $26 \mathrm{~S}$ proteasome is a large multi-subunit ATP dependent protease complex of approximately $2.6 \mathrm{MDa}$ that is responsible for the highly regulated proteolysis of proteins targeted for breakdown by ubiquitin conjugation [1]. The $26 \mathrm{~S}$ proteasome is a wellestablished target for cancer therapy and its deregulation is associated with neurodegenerative conditions such as Alzheimer's and Parkinson's diseases.

The $26 \mathrm{~S}$ proteasome consists of a $20 \mathrm{~S}$ core associated with two $19 \mathrm{~S}$ regulatory particles. Four stacked rings of seven highly homologous $\alpha$ or $\beta$ subunits, arranged as a $\alpha \beta \beta \alpha$ barrel-like structure, form the 20S core [2]. The proteolytic active sites, formed by the $N$-terminal Thr residues of the subunits $\beta 1, \beta 2$ and $\beta 5$, are located within the central cavity of the $20 \mathrm{~S}$ core barrel. The $19 \mathrm{~S}$ regulatory particles are located at each end of the $20 \mathrm{~S}$ core and are responsible for the recognition, unfolding and translocation of substrate proteins into the proteolytic core. Despite its fundamental

role in eukaryotic homeostasis, the structural organisation of the $26 \mathrm{~S}$ proteasome regulatory subunits and its overall functional and regulatory mechanisms are still largely unknown. High resolution structural information is required to address these issues.

We have determined the structure of the human $26 \mathrm{~S}$ proteasome by cryo-electron microscopy and single particle analysis (Figure 1). Secondary structure elements are clearly identified throughout the 3D map. With the improved resolution we can now describe in detail the conformational rearrangements on the $20 \mathrm{~S}$ core subunits induced by the binding of the regulatory particles, which we previously observed by analysis of negatively stained samples [3]. We can also directly identify the densities corresponding to the six ATPase subunits of the 19S regulatory particle, the Rpt subunits, and describe their structural relationship with the $20 \mathrm{~S}$ core. Finally, our map allows us to explore the organisation of the remaining non-ATPase subunits of the $19 \mathrm{~S}$ regulatory particle.

References:

[1] A.L. Goldberg, Nature, 426 (2003) 895.

[2] M. Unno et al., Structure, 10 (2002) 609.

[3] P.C. da Fonseca \& E.P. Morris, J. Biol. Chem., 283 (2008) 23305. 

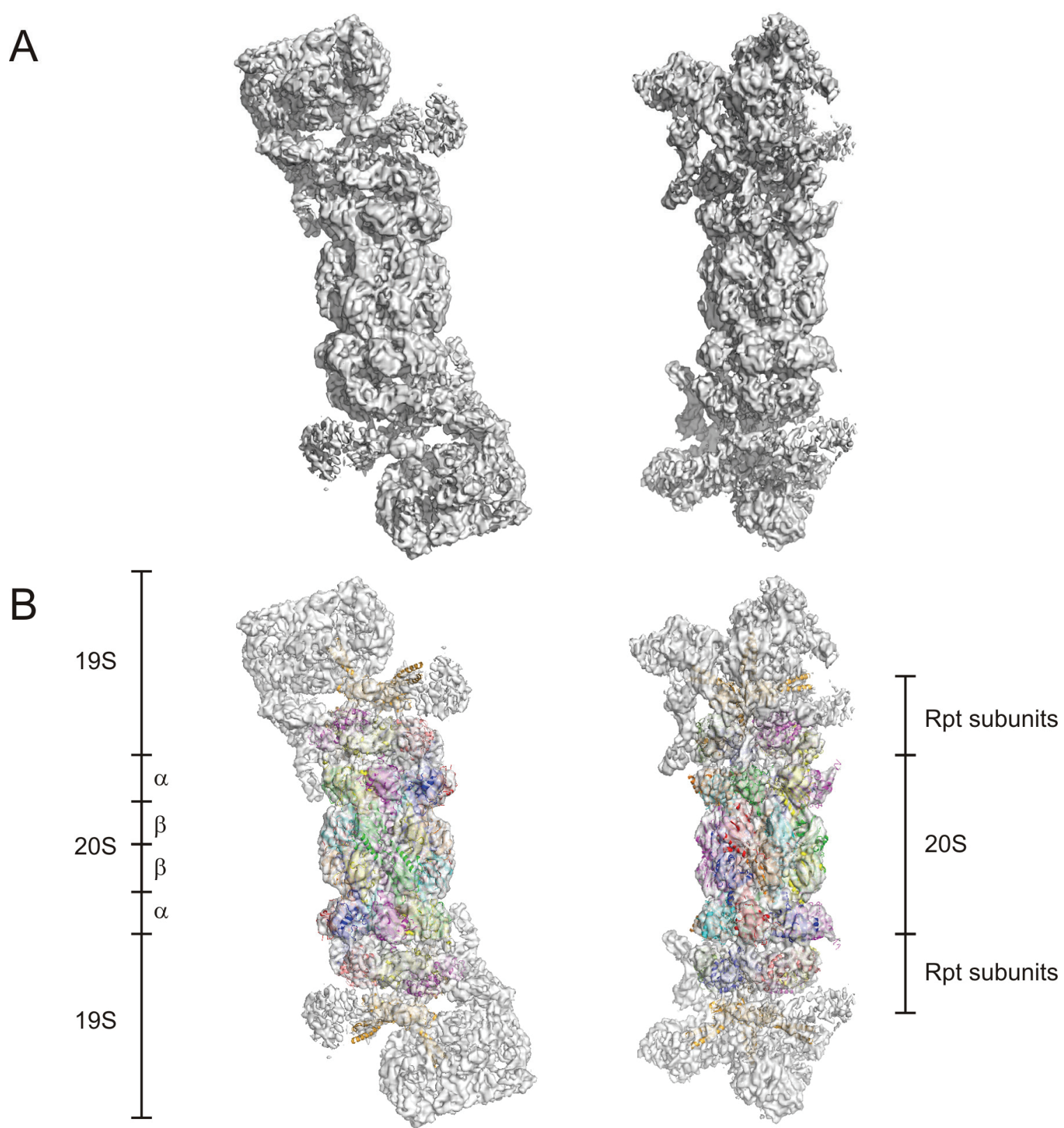

Figure 1. Structure of the $26 \mathrm{~S}$ proteasome. A: Surface representations of the 3D map of the double-capped 26S proteasome, viewed along (left) and normal (right) to its two-fold axis. B: The map of the $26 \mathrm{~S}$ proteasome is represented as transparent surfaces, oriented as in A, showing the docked coordinates for the 20S core (coordinates from the bovine $20 \mathrm{~S}$ core, PDB accession number 1IRU) and a model for the 19S Rpt subunits determined using the coordinates of the different sub-domains of their archaeal homologue, the PAN complex (PDB accession numbers 3H4M and 2WG5). The approximate location of proteasome components is indicated. 